\title{
The Electronic Structure of C.I. Pigment Red 209
}

\author{
Takatoshi Senju*, Jin Mizuguchi \\ Department of Applied Physics, Graduate School of Engineering \\ Yokohama National University \\ 79-5 Tokiwadai, Hodogaya-ku, 240-8501 Yokohama, Japan
}

\begin{abstract}
Pigment Red 209 (PR209) is a mixture of three kinds of isomeric dichloroquinacridones formed inevitably during the reaction process: 3,10-, 1,8- and 1,10-derivatives. PR209 has attracted attention for its yellowish shade of red, which is unusual for a QA pigment. To elucidate this, electronic characterization of PR209 has been carried out on the basis of the crystal structure. Among three derivatives, only 3,10-DClQA has been isolated as single crystals. X-ray structure analysis revealed that 3,10-DClQA crystallizes in the space group of $P-1$ and that there are chains of $\mathrm{NH} \cdots \mathrm{O}$ intermolecular hydrogen bonds along the [1-10] direction which constitute a two-dimensional hydrogen bond network. However, 3,10-DClQA is found to lack the yellowish component of PR209. The present component can be attributed to 1,8- and 1,10-DClQAs which are supposed to give
\end{abstract}


absorption bands at shorter wavelengths than 3,10-DClQA.

Keywords: Pigment red 209; 3,10-Dichloroquinacridone; Polymorph; Crystal structure;

Hydrogen-bonded pigment

* Corresponding author. Tel./Fax: +81 453394320.

E-mail address: tsenju@ynu.ac.jp (T. Senju) 


\section{Introduction}

Quinacridones (QAs) are industrially important hydrogen-bonded red pigments [1]. QA derivatives exhibit a wide range of red colors from vivid red to bluish red, depending upon the substituents as well as their crystal phases. Among these, Pigment Red 209 (abbreviated to PR209) has stimulated a certain amount of interest for its yellowish shade of red, which is unusual for a QA pigment. PR209 is a mixture of three kinds of isomeric dichloroquinacridones which are formed inevitably during the reaction process: 3,10-, 1,8- and 1,10-derivatives (Figs. 1 and 2) [2]. Among these, 3,10 -dichloroquinacridone $(3,10$-DClQA) is known to be the major component, and the ratio of these isomers depends on reaction conditions and starting materials [2].

In the present paper, electronic characterization of PR209 has been carried out on the basis of the crystal structure in order to clarify the yellowish component in PR209. We will characterize the electronic structure of PR209 with special attention to the excitonic interactions in accordance with our previous reports in consideration of the molecular arrangement [3-8].

\section{Experimental Section}

\subsection{Materials and crystal growth}


PR209 was obtained from Dainippon Ink and Chemicals, Inc. The samples were purified by sublimation, using a two-zone furnace [9]. Single crystals were then grown from the vapor phase at about $743 \mathrm{~K}$. After $24 \mathrm{~h}$, a number of red needle-shaped single crystals were obtained.

\subsection{Equipment and Measurements}

Solution spectrum in dimethyl sulfoxide (DMSO) was recorded on a UV-2400PC spectrophotometer (Shimadzu). Diffuse reflectance spectrum on powders was recorded using the same equipment together with an ISR-240A integrating sphere attachment from Shimadzu. Reflection spectra on single crystals were measured by means of a UMSP80 microscope-spectrophotometer (Carl Zeiss). An Epiplan Pol $(\times 8)$ objective was used together with a Nicol-type polarizer. Reflectivities were corrected relative to the reflection standard of silicon carbide.

\subsection{Molecular orbital calculations}

Semi-empirical molecular orbital (MO) calculations were performed using a Quantum CAChe ver. 3.2 program package [10] which includes MOPAC ver. 94.10 and the ZINDO programs [11]. Geometries were optimized using the AM1 Hamiltonian [12]. 
Spectroscopic calculations were then made on the optimized geometries using the INDO/S Hamiltonian [13] including 196 singly-excited configuration interactions composed of 14 occupied and 14 unoccupied MOs.

\section{Results and Discussion}

\subsection{Crystal Structure of 3,10-DClQA}

Single crystals were obtained by sublimation of PR209 which is composed of 3,10-, 1,8- and 1,10-derivatives. However, the single crystals were found to be 3,10-DClQA as revealed form the structure analysis. Table 1 details the crystallographic parameters [14]. Fig. 3 shows the ORTEP plot of the molecule. The molecule has inversion symmetry. The quinacridone skeleton is entirely planar as indicated by the root mean square deviation of $c a .0 .02 \AA$ from the least-squares plane of the rings defined by atoms C1-C10 and N1. However, the carbonyl O atom deviates shortly (0.140(3) Å) from QA skeleton towards the $\mathrm{NH}$ group of the neighboring molecule probably due to the formation of the $\mathrm{NH}^{\cdots} \mathrm{O}$ intermolecular hydrogen bond. This tendency is found in the red phase of 2,9-dichloroquinacridone [15] and 2,9-dimethylquinacridone [16]. As shown in Fig. 4(a), there are chains of $\mathrm{NH}^{\cdots} \mathrm{O}$ intermolecular hydrogen bonds along the $\left[\begin{array}{lll}1 & -1 & 0\end{array}\right]$ direction. One molecule is bonded to two neighboring molecules through 
four hydrogen bonds. The $\mathrm{H} / \mathrm{O}$ and N/O distances in the hydrogen bond are $2.12 \AA$ and 2.873(4) $\AA$, respectively, and the angle formed by the N, $\mathrm{H}$ and $\mathrm{O}$ atoms is $159.4^{\circ}$. This suggests the $\mathrm{NH} \cdots \mathrm{O}$ hydrogen bond in 3,10 -DClQA is quite strong. Furthermore, there is a small step of $c a .0 .55 \AA$ between the two molecular planes of the hydrogen-bonded molecules as shown in Fig. 4(b). These features are quite similar to those of the red phase of 2,9-dichloroquinacridone [15] and 2,9-dimethylquinacridone [16].

\subsection{Characterization of PR209 in Solution}

Table 2 lists the absorption bands and oscillator strength for 1,8-, 1,10- and 3,10-DClQAs calculated by semi-empirical MO calculations. The absorption wavelengths are $371 \mathrm{~nm}$ for 1,8- and 1,10-DClQAs and $372 \mathrm{~nm}$ for 3,10-DClQA, suggesting that all the isomers give nearly the same solution spectra. It is also to be noted that all of these calculated electronic transitions are the HOMO/LUMO $\pi-\pi^{*}$ transition, and this is the only one electronic transition which is expected to appear in the visible region. The direction of the transition dipole is perpendicular to the long-molecular axis as shown in Fig. 1.

Fig. 5 shows the solution spectrum of PR209 in dimethylsulfoxide (DMSO). A progression of the absorption bands is observed, which starts from $c a .523 \mathrm{~nm}$ toward 
shorter wavelengths. Furthermore, the absorption edge of the longest-wavelength band is quite steep and all bands are almost equally spaced. On the basis of these characteristics, the absorption bands are assigned to the $0-0,0-1$ and $0-2$ bands as designated in Fig. 5, indicating that one single electronic transition is coupled with vibrational transitions.

\subsection{Characterization of 3,10-DClQA in Single Crystals}

\subsubsection{Polarized reflection spectra measured on single crystals}

Fig. 6 shows the polarized reflection spectra measured on the (001) plane of single crystals of 3,10-DClQA together with its projection of the crystal structure. A prominent reflection band appears around $558 \mathrm{~nm}$ together with two small bands around 484 and $518 \mathrm{~nm}$ for polarization parallel to the direction of the hydrogen bond (Fig. 6(b)). On the other hand, these bands are completely quenched for polarization perpendicular to this direction. These results clearly indicate that the direction of the transition dipole points along the intermolecular hydrogen bond in accord with the direction as deduced from MO calculations and that all the reflection bands belong to one single $\pi-\pi^{*}$ electronic transition. It is also important to note that the spectral shape in the solid state

(Fig. 6(a)) is quite similar to that of the solution (Fig. 5) and the number of the 
absorption bands are the same. Therefore, one-to-one correspondence of the absorption bands between the solution and solid state spectra is possible. This indicates that the molecular nature is well preserved even in the solid state and that the solid state spectrum can be pictured as a merely bathochromically-displaced spectrum of the solution one, on going from solution to the solid state.

As for the band around $484 \mathrm{~nm}$, the intensity of this band is two small for lending a yellowish component of PR209, suggesting that the present shade is due to 1,8 - and 1,10-DClQA.

\subsubsection{Bathochromic shift upon crystallization}

The bathochromic shifts upon crystallization is often observed in QA compounds and is usually interpreted as arising from interactions between transition dipoles [3]. The $\mathrm{NH} \cdots \mathrm{O}$ intermolecular hydrogen bonds between the $\mathrm{NH}$ group of one molecule and the $\mathrm{O}$ atom of the neighboring one aligns the two molecules in a fashion "head-to-tail" which induces the maximum bathochromic shift. The present interaction is typical of dyestuff or pigment systems, in which the absorption coefficient of the component molecule is high and the molecules are periodically arranged [3-8]. The outline is described below. 
The interaction energy $\left(\Delta E_{\text {exciton }}\right)$ is given by the dipole-dipole equation $[17,18]$ : $\Delta E_{\text {exciton }}=|\mu|^{2}\left(1-3 \cos ^{2} \theta\right) / r^{3}$, where the transition dipole is denoted by $\mu$, the distance and angle between two transition dipoles by $r$ and $\theta$, respectively. As evident from the present equation, the overall shift energy is determined by the strength of the interneighbor coupling $\left(|\mu|^{2}\right)$ (i.e. proportional to the absorption coefficient of the molecule) as well as on the mutual relative orientation of the transition dipoles in molecular assemblies. That is, the term $\left(1-3 \cos ^{2} \theta\right) / r^{3}$ determines the geometrical relationship of transition dipoles correlated with the crystal structure. Since this term falls off as the inverse cube of distance, most of the interaction would come from the nearest neighbors. The bathochromic or hypsochromic shift depends on the critical angle of $\theta=54.7^{\circ}$ (i.e. $\Delta E_{\text {exciton }}=0$ ), below which the former will result and above which the latter will be the case. The maximum bathochromic shift arises when the transition dipoles are arranged in a fashion "head-to-tail."

As seen from Fig. 6(b), the $\mathrm{NH} \cdots \mathrm{O}$ intermolecular hydrogen bond aligns the molecules nearly in a "head-to-tail" fashion (i.e. $\theta \approx 0$ ) which induces the maximum bathochromic dispacement. Furthermore, the molar extinction coefficient (i.e. proportional to $|\mu|^{2}$ ) of the component molecule is as high as about 14000 (Fig. 5). In addition, the center-to-center distance between two transition dipoles is relatively short 
(6.92 $\AA$ ). Because of these parameters, a large bathochromic shift is expected to occur in 3,10-DClQA.

\subsection{Characterization of PR209 in the Solid State}

\subsubsection{Powder X-ray diffraction diagrams}

Fig. 7 shows the powder X-ray diffraction diagram for PR209 and the simulated diffraction diagram of 3,10-DClQA based upon the structure analysis described in section 3.1. Apparently, both diagrams are strikingly different, and only a few peaks can be assigned to 3,10-DClQA. The unassigned peaks may be attributed to other derivatives other than 3,10-DClQA.

\subsubsection{Diffuse reflectance spectrum}

Fig. 8 shows the diffuse reflectance spectrum measured on powdered PR209. Three characteristic bands appear in the visible region $(540,520$, and $475 \mathrm{~nm})$, among which the band around $520 \mathrm{~nm}$ is the strongest. The absorption shoulder around $475 \mathrm{~nm}$ is evidently responsible for the yellowish component of PR209. The spectral shape as well as the position and intensity of the absorption bands are appreciably different from those in the polarized reflection spectra of 3,10-DClQA (Fig. 6(a)). This clearly indicates that 
PR209 includes additional compounds other than 3,10-DC1QA.

\subsubsection{Yellowish shade in PR209}

One of the appealing features of PR209 is the yellowish shade of red, which is unusual for a quinacridone. The yellowish shade means that there is an absorption around 480 $\mathrm{nm}$, and this is clearly observed in PR209 (Fig. 8); whereas this component is missing in 3,10-DCIQA (Fig. 6(a)). Furthermore, the diffraction diagram of powdered PR209 differs significantly from that of the simulated diagram based upon the X-ray structure analysis of 3,10-DClQA. In this connection, we propose the following explanation. This is a speculation, but it is of great help for developing an understanding of the mechanism of the yellowish component. The discussion here focuses on how the yellowish component appears from 1,8- and 1,10-derivatives (Fig. 1). The Cl-substituent at the 1-position in these derivatives is likely to hinder or weaken the formation of the $\mathrm{NH}^{\cdots} \mathrm{O}$ hydrogen bond due to steric hindrance in comparison with that of the 3-position in 3,10-DClQA. This exerts a profound influence on the geometry of the hydrogen bond which governs directly the extent of the bathochromic shift due to excitonic interactions. The weakening of the $\mathrm{NH}^{\cdots} \mathrm{O}$ hydrogen bond caused by the steric hindrance induces a smaller bathochromic shift as compared with that of 3,10 -DClQA 
(Fig. 6(a)). Then, the whole spectrum shown in Fig. 6(a) is displaced toward shorter wavelengths, for example, by about $20 \mathrm{~nm}$ so that the longest-wavelength band occurs around $520 \mathrm{~nm}$. The superposition of this displaced spectrum (assumed for 1,8- and 1,10-derivatives) and the spectrum of 3,10-DClQA (Fig. 6(a)) gives approximately Fig. 8. The band around $480 \mathrm{~nm}$ is due to the second-strongest band of the assumed spectrum of 1,8- and 1,10-derivatives.

\section{Conclusions}

Electronic structure of PR209 has been investigated on the basis of the crystal structure.

The conclusions can be summarized as follows.

1. Single crystals of 3,10-DClQA have been isolated when PR209 was sublimed under vacuum. $3,10-\mathrm{DClQA}$ is found to crystallize in the space group of $P-1$. There are chains of $\mathrm{NH} \cdots \mathrm{O}$ hydrogen bonds along the $[1-10]$ direction, and this constitutes a two-dimensional network.

2. A large bathochromic shift of 3,10-DClQA is observed upon crystallization. This is interpreted as arising from interactions between transition dipoles arranged nearly in a "head-to-tail" fashion by means of $\mathrm{NH} \cdots \mathrm{O}$ hydrogen bonds.

3. 3,10-DClQA is found to lack the absorption component around $480 \mathrm{~nm}$ that lends a 
yellowish-red color of PR209. This component is presumably provided by 1,8 - and 1,10-DClQA whose absorption bands are supposed to appear at shorter wavelengths than those of 3,10-DClQA. This is caused by the weakening of the $\mathrm{NH} \cdots \mathrm{O}$ hydrogen bonds due to the steric hindrance of the Cl-substituent located at the 1-position of 1,8- and 1,10-derivatives. 


\section{References}

[1] Herbst W, Hunger K. Industrial Organic Pigments, 2nd ed. Weinheim, Germany:VCH, 1997. p. 454-474.

[2] Altiparmakian RH, Bohler H, Kaul BL, Kehrer F. Quinacridones. Structure and mechanism of formation. Helv Chim Acta 1972;55(1):85-100.

[3] Mizuguchi J, Senju T. Solution and solid-state spectra of quinacridone derivatives as viewed from the intermolecular hydrogen bond, J Phys Chem B 2006;110(39):19154 19161.

[4] Mizuguchi J, Shikamori H. Spectral and crystallographic coincidence in a mixed crystal of two components and a crystal of their hybrid component in pyrrolopyrrole pigments, J Phys Chem B 2004;108(7):2154-2161.

[5] Senju T, Mizuguchi J. Electronic Structure of Thiazine-Indigo Pigment on the Basis of the Crystal Structure, J Phys Chem B 2005;109(16):7649-7653.

[6] Mizuguchi J, Tojo K. Electronic structure of perylene pigments as viewed from the crystal structure and excitonic interactions, J Phys Chem B 2002;106(4):767-772.

[7] Mizuguchi J. Crystal structure and electronic characterization of trans and cis perinone pigments, J Phys Chem B 2004;108(26):8926-8930.

[8] Endo A, Matsumoto S, Mizuguchi J. Interpretation of the near-IR absorption of magnesium phthalocyanine comlexes in terms of molecular distortion and exciton 
coupling effects, J Phys Chem A 1999;103:8193-8199.

[9] Mizuguchi J. An improved method for purification of $\beta$-copperphthalocyanine, Krist Tech $1981 ; 16: 695-700$.

[10] Quantum CAChe, version 3.2. Kanagawa, Japan: Fujitsu, Ltd, 1999.

[11] Ridley J, Zerner M. Intermediate neglect of differential overlap (INDO) technique for spectroscopy. Pyrrole and the azines. Theor Chim Acta 1973;32(2):111-134.

[12] Dewar MJS, Zoebisch EG, Healy EF, Stewart JJP. Development and use of quantum mechanical molecular models. 76. AM1: a new general purpose quantum mechanical molecular model. J Am Chem Soc 1985;107(13):3902-3909.

[13] Kotzian M, Roesch N, Zerner MC. Intermediate neglect of differential overlap spectroscopic studies on lanthanide complexes. I. Spectroscopic parametrization and application to diatomic lanthanide oxides $\mathrm{LnO}(\mathrm{Ln}=$ lanthanum, cerium, gadolinium, and lutetium). Theor Chim Acta 1992;81(4-5):201-222.

[14] Senju $\quad$ T, Hoki T, 3,10-Dichloro-5,12-dihydroquino[2,3-b]acridine-7,14-dione. Acta Cryst 2006;E62(1):o261-o263.

[15] Senju T, Nishimura N, Hoki T, Mizuguchi J. 2,9-Dichloro-5,12-dihydroquino[2,3-b]acridine-7,14-dione (red phase). Acta Cryst 
2005;E61(8):o2596-o2598.

[16] Mizuguchi J, Senju T, Sakai M. Crystal structure of 5,12-dihydro-2, 9-dimethylquino[2,3-b]acridine-7,14-dione, $Z$ Krist New Cryst Struct $2002 ; 217: 525-526$.

[17] Kasha M. Molecular Excitons in Small Aggregates. In: Bartolo BD, editor. NATO Advanced Study Institute Series, Series B, Vol. 12. New York: Plenum Press, 1976. p. $337-363$.

[18] Craig DP, Walmsley, SH. Excitons in Molecular Crystals: theory and applications.

New York: W. A. Benjamin, Inc., 1968. 


\section{Figure Captions}

Fig. 1. Molecular structure of 3,10-DClQA. The dotted line denotes the transition dipole $(\mu)$ as deduced from MO calculations.

Fig. 2. Reaction scheme for the preparation of 3,10-DClQA.

Fig. 3. ORTEP plot for the molecule of 3,10-DCIQA.

Fig. 4. (a) Top view of the two hydrogen-bonded molecules. The dotted line denotes intermolecular $\mathrm{N}-\mathrm{H} \cdots \mathrm{O}$ hydrogen bonds, and (b) side view of the two hydrogen-bonded molecules, showing a small step of $c a .0 .55 \AA$.

Fig. 5. Solution spectrum of PR209 in DMSO.

Fig. 6. (a) Polarized reflection spectra measured on the (001) plane of single crystals of 3,10-DClQA, and (b) projection of the crystal structure onto the (001) plane. The thick solid line denotes the transition dipole $(\mu)$, while the intermolecular hydrogen bonds are designated by dotted lines.

Fig. 7. X-ray diffraction diagrams: (a) powdered PR209 and (b) simulated diffraction diagram of 3,10-DClQA based upon the structure analysis.

Fig. 8. Diffuse reflectance spectrum measured on powdered PR209. 


\section{Tables}

Table 1

Crystallographic parameters of 3,10-DClQA

\begin{tabular}{cc}
\hline Molecular formula & $\mathrm{C}_{20} \mathrm{H}_{10} \mathrm{Cl}_{2} \mathrm{~N}_{2} \mathrm{O}_{2}$ \\
Molecular weight & 381.20 \\
Crystal system & triclinic \\
Space group & $P-1$ \\
$Z$ & 1 \\
$a(\AA)$ & $3.7635(13)$ \\
$b(\AA)$ & $5.853(2)$ \\
$c(\AA)$ & $16.746(6)$ \\
$\alpha\left(^{\circ}\right)$ & $85.20(2)$ \\
$\beta\left(^{\circ}\right)$ & $83.79(2)$ \\
$\gamma\left({ }^{\circ}\right)$ & $89.32(2)$ \\
$V\left(\AA^{3}\right)$ & $365.4(2)$ \\
$R_{1}$ & 0.057 \\
$D_{\text {calcd }}\left(\mathrm{Mg} \mathrm{m}^{-3}\right)$ & 1.732 \\
$T(\mathrm{~K})$ & $93(1)$ \\
\hline
\end{tabular}

Table 2

Calculated absorption bands and oscillator strengths for 1,8-, 1,10- and 3,10-DClQAs

\begin{tabular}{lcc}
\hline Compound & $\lambda_{\max }(\mathrm{nm})$ & $\begin{array}{c}\text { Oscillator } \\
\text { strength }(f)\end{array}$ \\
\hline 1,8-DClQA & 371 & 0.29 \\
1,10-DClQA & 371 & 0.28 \\
3,10-DClQA & 372 & 0.28 \\
\hline
\end{tabular}




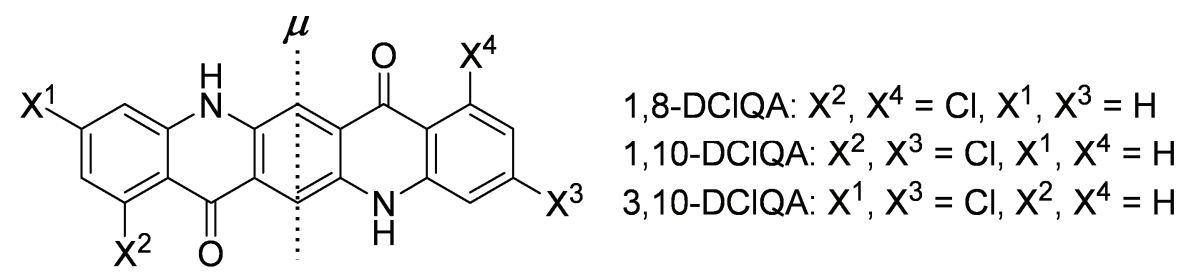

Fig. 1. Molecular structure of 3,10-DClQA. The dotted line denotes the transition dipole $(\mu)$ as deduced from MO calculations.<smiles>[R]OC(=O)C1=C(Nc2cccc(Cl)c2)CC(C(=O)O[R2])=C(Nc2cccc(Cl)c2)C1</smiles>

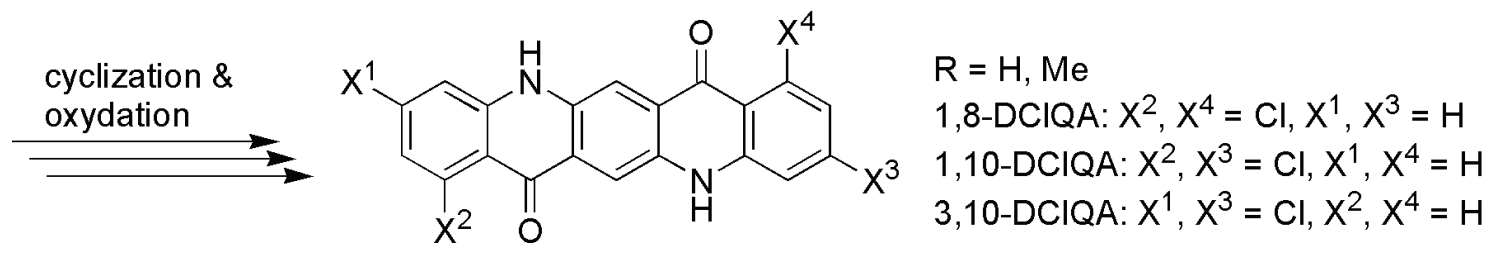

Fig. 2. Reaction scheme for the preparation of 3,10-DClQA. 


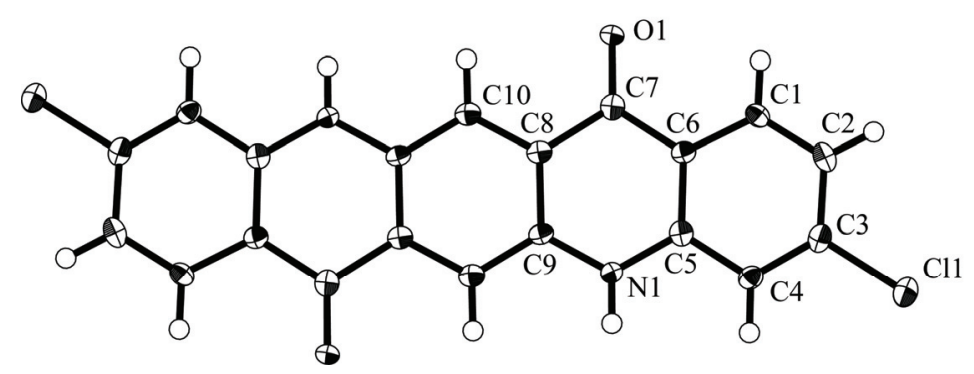

Fig. 3. ORTEP plot for the molecule of 3,10-DCIQA.

(a)

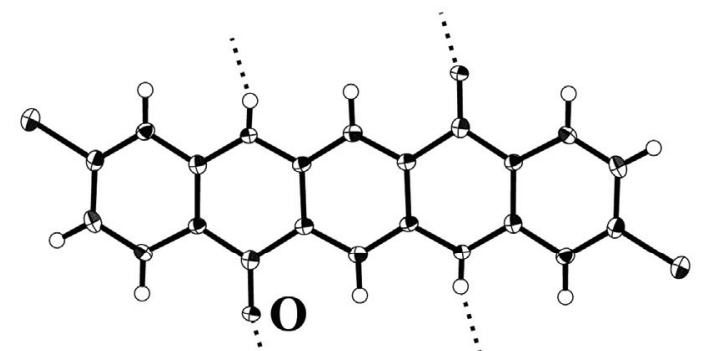

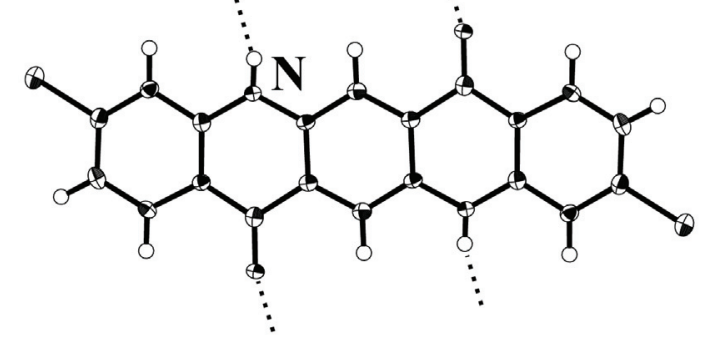

(b)

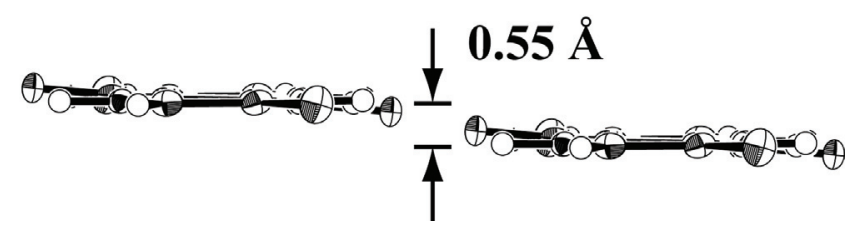

Fig. 4. (a) Top view of the two hydrogen-bonded molecules. The dotted line denotes intermolecular $\mathrm{N}-\mathrm{H} \cdots \mathrm{O}$ hydrogen bonds, and (b) side view of the two hydrogen-bonded molecules, showing a small step of $c a .0 .55 \AA$. 


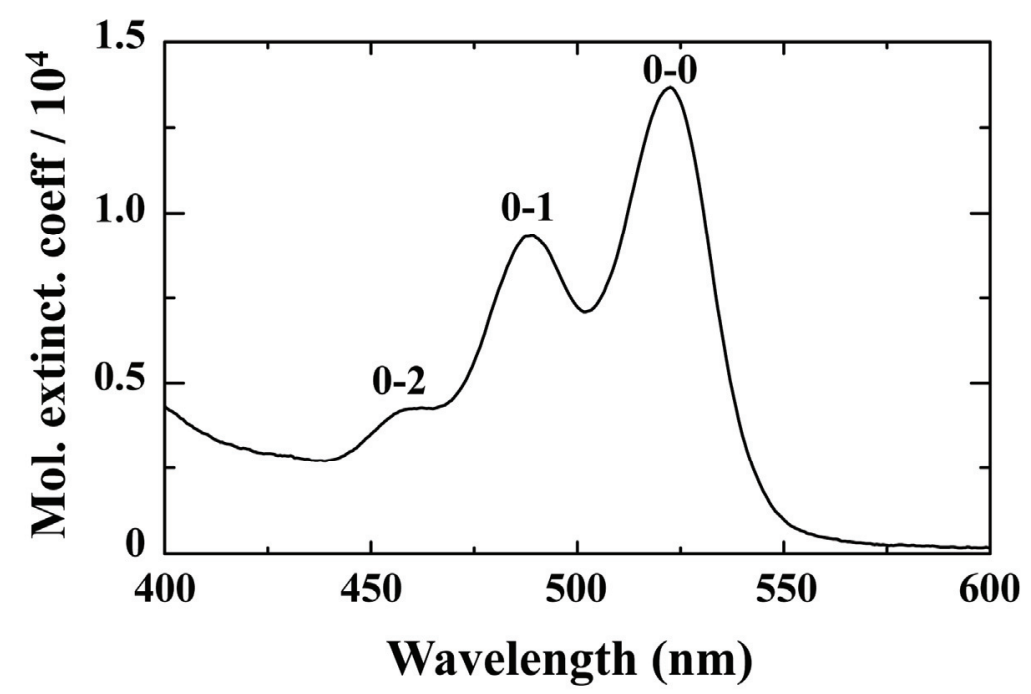

Fig. 5. Solution spectrum of PR209 in DMSO. 
(a)

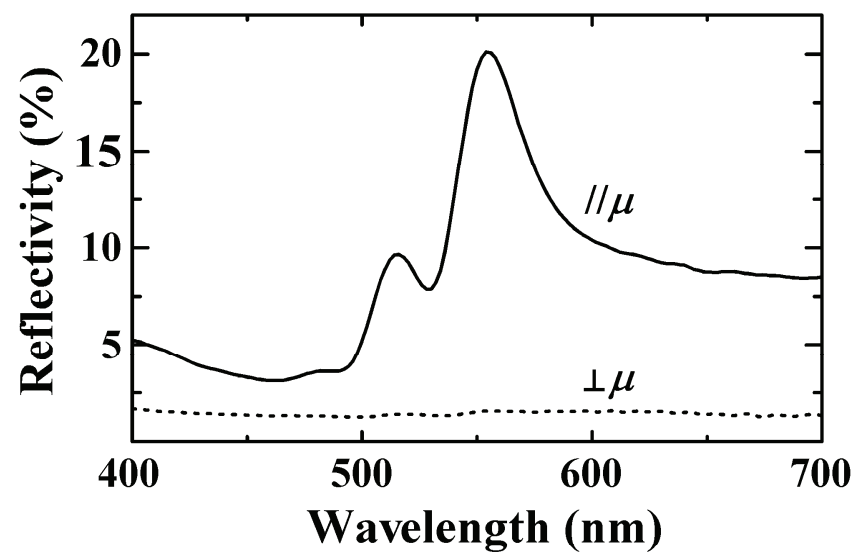

(b)

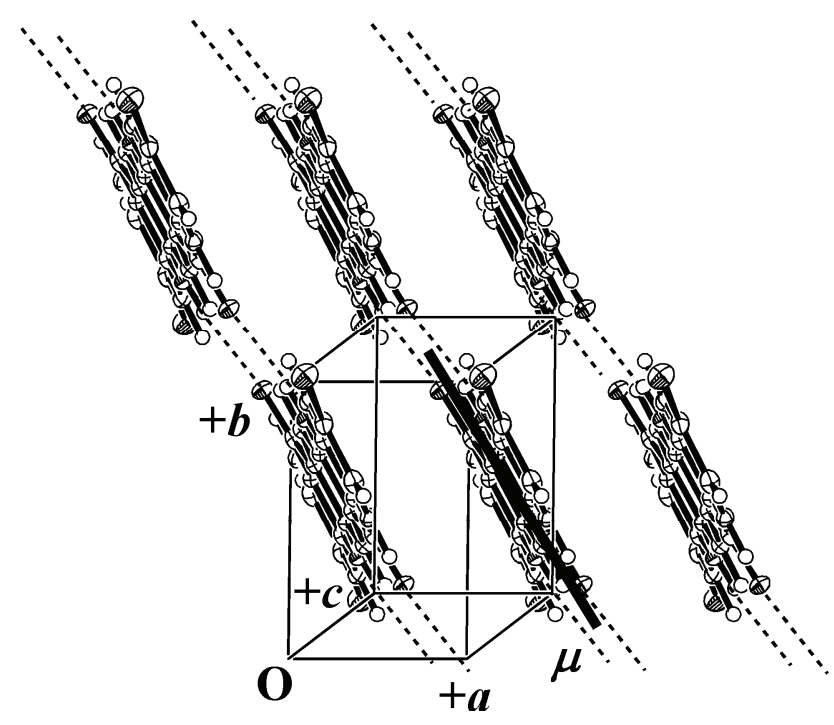

Fig. 6. (a) Polarized reflection spectra measured on the (001) plane of single crystals of 3,10-DClQA, and (b) projection of the crystal structure onto the (001) plane. The thick solid line denotes the transition dipole $(\mu)$, while the intermolecular hydrogen bonds are designated by dotted lines. 


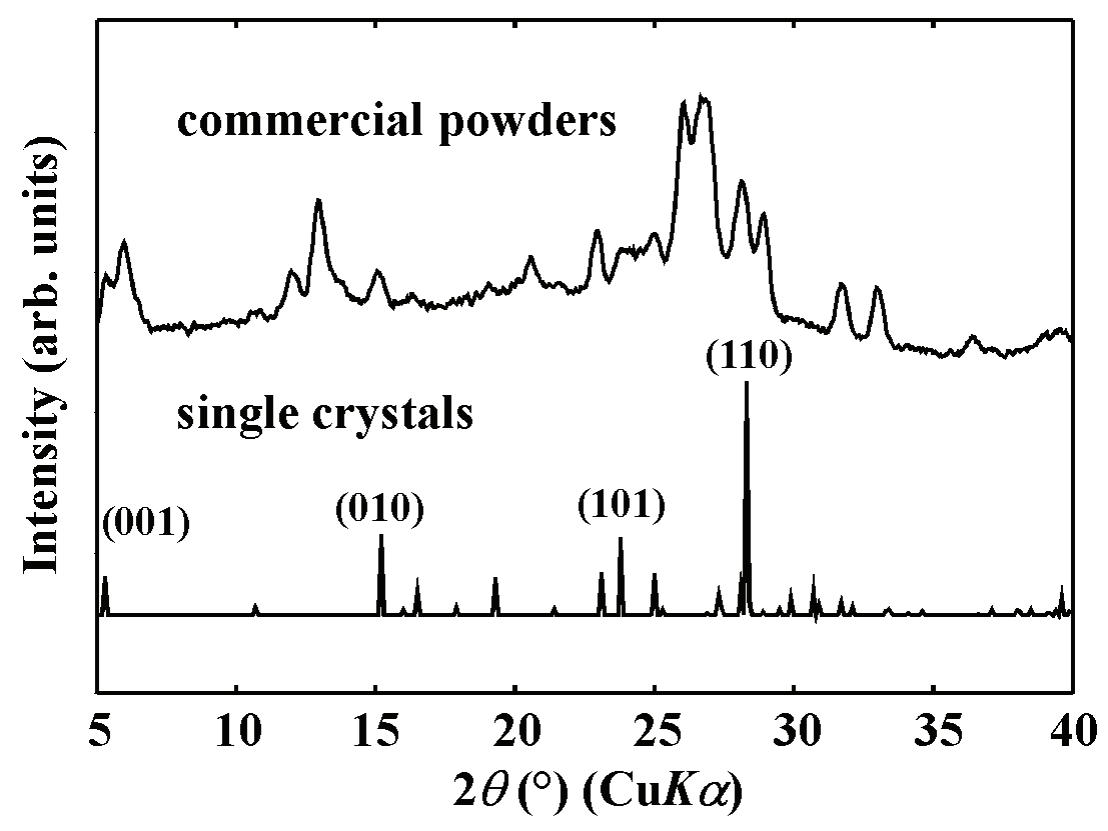

Fig. 7. X-ray diffraction diagrams: (a) powdered PR209 and (b) simulated diffraction diagram of 3,10-DClQA based upon the structure analysis.

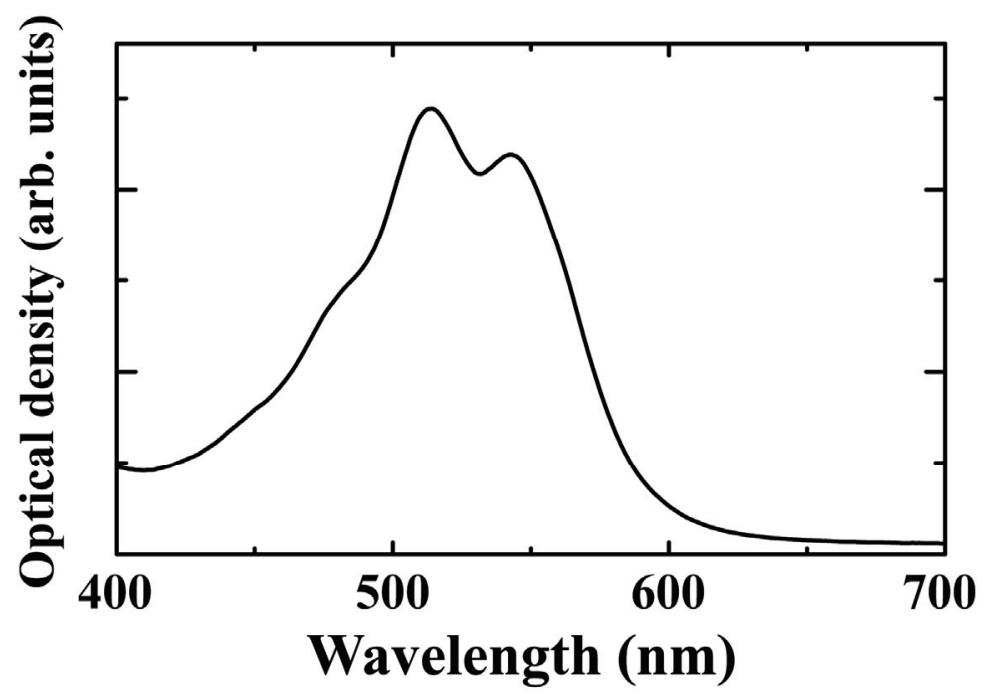

Fig. 8. Diffuse reflectance spectrum measured on powdered PR209. 